\title{
Thermomechanical Behavior of High Performance Epoxy/Organoclay Nanocomposites
}

\author{
Artur Soares Cavalcanti Leal, ${ }^{1}$ Carlos Jose de Araújo, ${ }^{2}$ \\ Antônio Gilson de Barbosa Lima, ${ }^{3}$ and Suédina Maria Lima Silva ${ }^{3}$ \\ ${ }^{1}$ Post-Graduate Program in Process Engineering, Federal University of Campina Grande, Avenue Aprígio Veloso 882, \\ 58429-900 Campina Grande, PB, Brazil \\ ${ }^{2}$ Mecanichal Engineering Department, Federal University of Campina Grande, Avenue Aprígio Veloso 882, \\ 58429-900 Campina Grande, PB, Brazil \\ ${ }^{3}$ Materials Engineering Department, Federal University of Campina Grande, Avenue Aprígio Veloso 882, \\ 58429-900 Campina Grande, PB, Brazil
}

Correspondence should be addressed to Artur Soares Cavalcanti Leal; arturcleal@yahoo.com.br

Received 28 February 2014; Accepted 11 May 2014; Published 5 June 2014

Academic Editor: J. M. P. Q. Delgado

Copyright (C) 2014 Artur Soares Cavalcanti Leal et al. This is an open access article distributed under the Creative Commons Attribution License, which permits unrestricted use, distribution, and reproduction in any medium, provided the original work is properly cited.

\begin{abstract}
Nanocomposites of epoxy resin containing bentonite clay were fabricated to evaluate the thermomechanical behavior during heating. The epoxy resin system studied was prepared using bifunctional diglycidyl ether of bisphenol A (DGEBA), crosslinking agent diaminodiphenylsulfone (DDS), and diethylenetriamine (DETA). The purified bentonite organoclay (APOC) was used in all experiments. The formation of nanocomposite was confirmed by X-ray diffraction analysis. Specimens of the fabricated nanocomposites were characterized by dynamic mechanical analysis (DMA). According to the DMA results a significant increase in glass transition temperature and storage modulus was evidenced when $1 \mathrm{phr}$ of clay is added to epoxy resin.
\end{abstract}

\section{Introduction}

Polymeric nanocomposites are hybrid materials where the dispersed phase has nanodimensions not exceeding $10 \mathrm{~nm}$ [1]. Similar to traditional composites, the continuous phase is an organic matrix (polymer) in which inorganic particles are dispersed in nanodimensions. By presenting significant gains in thermal, mechanical, and thermomechanical properties, barrier to gases and liquids, and flame retardance, nanocomposites of polymer/clay have attracted considerable attention in recent years [2].

The thermal behavior of epoxy systems depends on a number of factors such as type and concentration of the used components, mixing conditions, curing conditions, and postcure treatment. An increase in glass transition temperature $\left(T_{q}\right)$ of epoxy/clay nanocomposites was observed by Lan et al. [3], while Massam and Pinnavaia [4] observed a reduction in $T_{g}$ of the material. The increase in $T_{g}$ was attributed to the slowing of molecular motion due to the interaction of nanoparticles, while the decrease can be explained by disruption of the reticulated structure, or residual monomers polymerization.

Like most epoxy nanocomposites actually presenting an intercalated structure, it is not surprising that most studies have shown that there was a reduction in $T_{g}$. However, different concentrations of curing agent and various methods for obtaining these nanocomposites, as well as the type of clay used, directly influence the results. Based on this description it is evident that the preparation of polymeric nanocomposites does not always contribute to an increase in thermal stability of epoxy systems. Santos [5] in his work on the cure kinetics and thermal stability of epoxy systems showed that variations in the structure of the nanocomposite can produce contradictory results on the thermal stability. The author found that although he had a considerable gain in the mechanical properties of epoxy/clay nanocomposite, 
thermal properties were not improved. The thermal stability and glass transition temperature of these materials were lower than the epoxy system without nanoparticles dispersed.

An interesting characteristic that enables epoxy/clay nanocomposite to be used as a matrix for receiving a Ni-Ti shape memory alloy (SMA) is the thermal stability, which is directly influenced by the structure of nanocomposites. Exfoliated structures have better thermal stability in the range of transformation of SMA compared to nanocomposites with intercalated structures [6-10], and these results were observed in the results of dynamic mechanical analysis (DMA), for proportions of clay below $5 \%$ in mass.

The results, sometimes divergent of the thermomechanical properties of nanocomposites, are due to the fact that these properties are affected not only by the structure of nanocomposite, but also for many other factors, such as the crosslinking density, charge-matrix adhesion, reaction kinetics, and the method of determination of the thermal proprieties [11].

An effective method to study the thermal properties of nanocomposites is the dynamic mechanical analysis (DMA). In this work, the thermal behavior of a polymer matrix composed of one bifunctional crosslinking monomer diglycidyl ether of bisphenol A (DGEBA) and two different types of crosslinking agent, aliphatic amines diethylenetriamine (DETA) and aromatic amine diaminodiphenylsulfone (DDS), was evaluated in order to select it as polymeric matrix of better thermal stability.

In this context, this paper aims to apply the general technique of dynamic mechanical analysis (DMA) to evaluate the effect of curing agent and of the treatment of postcuring in the thermal properties of different systems. In order to select an adequate epoxy polymer matrix to be used in nanocomposites, the incorporation of $\mathrm{Ni}-\mathrm{Ti}$ alloy wire with shape memory effect is studied.

\section{Experimental Procedure}

2.1. Materials. Sodium bentonite purified (APOC), with cation exchange capacity (CEC) value of about $100 \mathrm{meq} / 100 \mathrm{~g}$, was purchased from Bentonit União Nordeste, Campina Grande, Paraíba State, Brazil. The preparation of organobentonite by ion exchange was carried out according to previously reported methods [12]; the organobentonite was modified with cetyl trimethyl ammonium bromide $\left(\mathrm{C}_{16} \mathrm{H}_{33}\left(\mathrm{CH}_{3}\right)_{3} \mathrm{NBr}\right)$. The used epoxy resin was the diglycidyl ether of bisphenol A (DGEBA) supplied by Silaex Química Ltda., São Paulo, São Paulo State, Brazil. The epoxide equivalent weight of epoxy resin was $182-192 \mathrm{~g} \mathrm{eq}^{-1}$. Aliphatic amines, diaminodiphenylsulfone (DDS) and diethylenetriamine (DETA), were used as curing agents for epoxy resin. The chemical structures of DDS and DETA epoxy resins are shown in Figure 1.

For the isothermal cure experiment, the epoxy resin and the curing agent are mixed in the stoichiometric ratio of 44 parts per hundred resin (phr) to curing agent DDS and to 36 phr to curing agent (DETA). The mixture was then mixed with $1 \%$ organobentonite powder.
TABLE 1: Specification of the polymer matrix studied.

\begin{tabular}{lccc}
\hline Component & Curing & Postcure & Coding \\
\hline DGEBA/DETA 7 hours $/ 110^{\circ} \mathrm{C}$ & $160^{\circ} \mathrm{C} / 2$ hours & DGEBA_DETApc \\
DGEBA/DETA 7 hours $/ 110^{\circ} \mathrm{C}$ & - & DGEBA_DETA \\
DGEBA/DDS & 5 hours $/ 160^{\circ} \mathrm{C}$ & $200^{\circ} \mathrm{C} / 2$ hours & DGEBA_DDSpc \\
DGEBA/DDS & 5 hours $/ 160^{\circ} \mathrm{C}$ & - & DGEBA_DDS \\
\hline
\end{tabular}

2.2. Synthesis of the Epoxy Resin. For the synthesis of epoxy resin using DGEBA/DDS (resin/hardener), a specific quantity $(40 \mathrm{~g})$ of DGEBA was placed in a Becker glass and heated in an oven at $110^{\circ} \mathrm{C}$ for 15 minutes and an amount equivalent to $44 \mathrm{phr}$ (parts per hundred resin) of DETA hardener was added. The mixture DGEBA/DDS was mixed mechanically at low rotation speed $(\sim 200 \mathrm{rpm})$ at temperature $90^{\circ} \mathrm{C}$ for 15 minutes before being inserted into the cast in a metallic mold, previously manufactured with rectangular cavities $125 \times$ $12.7 \times 3.2 \mathrm{~mm}$, corresponding to the shape of a flexion test specimens according to ASTM D790. The samples were kept at $160^{\circ} \mathrm{C}$ for a period of 5 hours. After this period, the epoxy resin was postcured at $200^{\circ} \mathrm{C}$ for 2 hours to obtain the maximum conversion.

The procedure used for the synthesis of DGEBA/DETA resin was similar to that used for DGEBA/DDS. However, in this case 36 phr of crosslinking agent was used (DETA). The resin was cured at $110^{\circ} \mathrm{C}$ for 7 hours and postcured at $135^{\circ} \mathrm{C}$ for 2 hours. The curing and postcure conditions as well as the codification of the epoxy systems studied are presented in Table 1.

2.3. Synthesis of the Nanocomposite. The epoxy resin DGEBA $(40 \mathrm{~g})$ was mixed with the desired amount of organophilic clay. The organoclay (325 mesh) was dispersed uniformly in epoxy monomer at $50^{\circ} \mathrm{C}$ and $200 \mathrm{rpm}$ for $1 \mathrm{~h}$. Then, the curing agent (DDS) was added into the epoxy/clay hybrid system and mixed thoroughly by stirring. The mixtures were cast into a mold. The samples were cured with DDS for $5 \mathrm{~h}$ and $160^{\circ} \mathrm{C}$ and postcured for $2 \mathrm{~h}$ at $200^{\circ} \mathrm{C}$. The procedure used in the synthesis of DGEBA/DETA was similar to that used for DGEBA/DDS. However, in this case the mixture DGEBA/DETA poured into the mold cavity was placed in an oven at $110^{\circ} \mathrm{C}$, maintained for a period of 7 hours, and postcured at $160^{\circ} \mathrm{C}$ for 2 hours. Conditions for healing and posttreatment, including the codes of the systems studied, are shown in Table 1.

2.4. Experimental Tests. In order to study the phase morphology, all samples were examined by DMA analysis, a powerful technique to study the relaxation and miscibility behavior of polymers and blends. The tangent to the peak of temperature, that is, $T_{g}$, can be used to evaluate miscibility and immiscibility of polymer blends [13]. Dynamic mechanical properties of nanocomposites and composites were measured with a TA Q800 dynamical mechanical analyzer (DMA) in fixed frequency mode at $1 \mathrm{~Hz}$ with $15 \mu \mathrm{m}$ oscillation amplitude, and temperature ranges from 30 to $250^{\circ} \mathrm{C}$ with variation of $5^{\circ} \mathrm{C} / \mathrm{min}$ loaded under single cantilever mode. The sample 


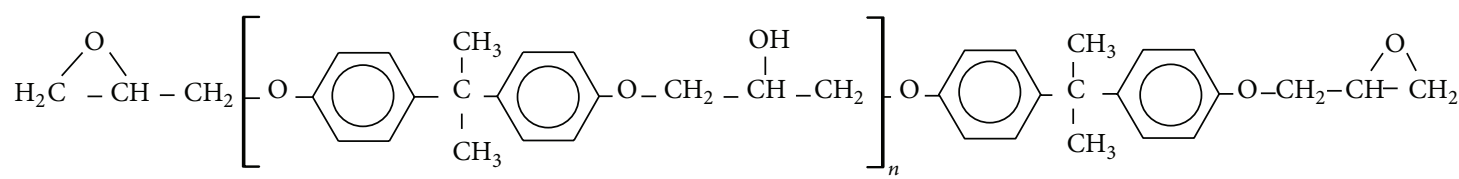

(a)

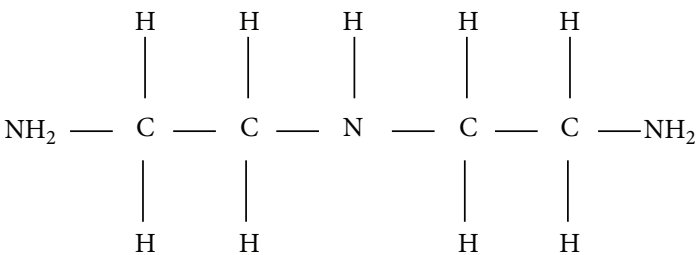

(b)

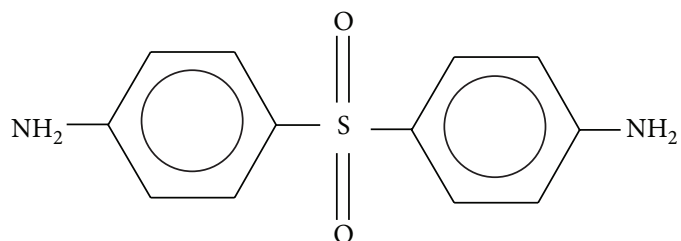

(c)

FIGURE 1: Chemical structures of epoxy resins. (a) DGEBA, (b) DETA, and (c) DDS.

size was $12.7 \times 3.47 \times 2 \mathrm{~mm}$. The glass transition temperature $\left(T_{g}\right)$ was determined from the peak of the $\tan \delta$ curve. Xray diffraction (XRD) analysis was performed on a Shimadzu XRD-6000 diffractometer with $\mathrm{Cu} K \alpha$ radiation $(\lambda=$ $0.154 \mathrm{~nm}, 40 \mathrm{kV}, 30 \mathrm{~mA}$ ) at room temperature. Diffraction was performed from $1.5^{\circ}$ to $12^{\circ}$ at a scan rate of $2^{\circ} / \mathrm{min}$ with a step size of $0.02^{\circ}$.

\section{Results and Discussion}

3.1. Thermomechanical Properties of the Epoxy Resin. The dynamic mechanical analysis (DMA) was performed to investigate the influence of the APOC concentration and postcuring treatment on the storage modulus and on the transition $(\tan \delta)$ of the nanocomposites. Figures 2(a) and 2(b) show the curves of damping $(\tan \delta)$ and storage modulus $E^{\prime}$ as a function of the temperature for epoxy systems cured with hardener DETA (EPX_DETA) and with hardener DDS (EPX_DDS), after having undergone postcuring treatment at different conditions (EPX_DETApc and EPX_DDSpc). A postcuring treatment in the systems evaluated at a temperature slightly above the $T_{g}$ of the material was perfomed, in order to obtain the maximum convection system.

It was evident (Figure 2 and Table 2) that the thermal behaviors of epoxy resin diglycidyl ether of bisphenol A (DGEBA) are affected by the type of curing agent (hardener) and postcure treatment. The epoxy resin cured with DDS hardeners (EPX_DDS system) showed higher values of $T_{g}$ (determined by the value of the peak of $\tan \delta$ ) than that cured with hardener DETA (EPX_DETA). When these systems were subjected to the treatment of postcure, EPX_DETApc and EPX_DDSpc, the increase in the value of $T_{g}$ was even more significant. According to Jones [14], the $\stackrel{g}{g}_{g}$ changes with the structure of cured resin and thermal history of the sample. These authors showed that even after the complete reaction of epoxy groups, that is, degree of conversion $\left(X_{e}\right)$ of approximately 99\%, an increase in $T_{g}$ was observed. For long times of postcuring, the $T_{g}$ is still increasing, although all epoxy groups have reacted. The reason for this behavior is due to variations in the chemical structure of the lattice that occur after essentially all epoxy groups have reacted; moreover,
TABLE 2: Cure and postcure temperature peak factor and the glass transition temperature $T_{g}$ for different epoxy systems.

\begin{tabular}{lccc}
\hline Epoxy system & $\begin{array}{c}\text { Cure and postcure } \\
\text { temperature }\end{array}$ & Peak factor $(\Gamma)$ & $T_{g}\left({ }^{\circ} \mathrm{C}\right)$ \\
\hline EPX_DETA & $110^{\circ} \mathrm{C} / 7 \mathrm{~h}$ & 0.1706 & 126.5 \\
EPX_DDS & $160^{\circ} \mathrm{C} / 5 \mathrm{~h}$ & 0.2878 & 175.1 \\
EPX_DETApc & $130^{\circ} \mathrm{C} / 7 \mathrm{~h} / 2 \mathrm{~h}$ & 0.7590 & 140.5 \\
EPX_DDSpc & $200^{\circ} \mathrm{C} / 5 \mathrm{~h} / 2 \mathrm{~h}$ & 0.1690 & 212.2 \\
\hline
\end{tabular}

there are considerable variations in density and variations in $T_{g}$ for times of healing or postcure when the extent of reaction is kept essentially constant, that is, $X_{e} \sim 0.99$. These reactions allowed restricting the molecular mobility in the region of glass transition and, therefore, considerable increases in the values of $T_{g}$ are observed [15].

As shown in Figure 2(a) the amplitude and the width of the peak $\tan \delta$ were modified when the epoxy systems were undergoing postcure treatment, especially for the system cured with DDS. According to literature [13], the peak factor ( $\Gamma)$ (Table 1 ), defined as the full width at half maximum of the $\tan \delta$ divided by its height, can be qualitatively used to evaluate the homogeneity of epoxy network. A higher peak factor for nanocomposite indicates a lower cross-link density and greater heterogeneity, which suggest intercalation of the epoxy network into the layers. Then, probably the homogeneity of the reticulation system for the epoxy cured with DDS was higher than for the system cured with DETA.

3.2. X-Ray Diffraction Analysis. Figure 3 shows X-ray diffraction patterns of the clay APOC and systems cured with epoxy curing agents DETA and DDS containing 1 parts per hundred resin (phr) of organoclay APOC (EPX_DETA.APOC and EPX_DDS.APOC) postcured. The clay showed the peak (001) at $2 \theta=4.75^{\circ}$ corresponding to a basal spacing of $1.85 \mathrm{~nm}$, which corresponds to an interlayer spacing of the clay. The absence of peak (001) for EPX_DETA.APOC and EPX_DDS.APOC systems indicates the penetration of resin in the galleries of the clay and the formation of predominantly 


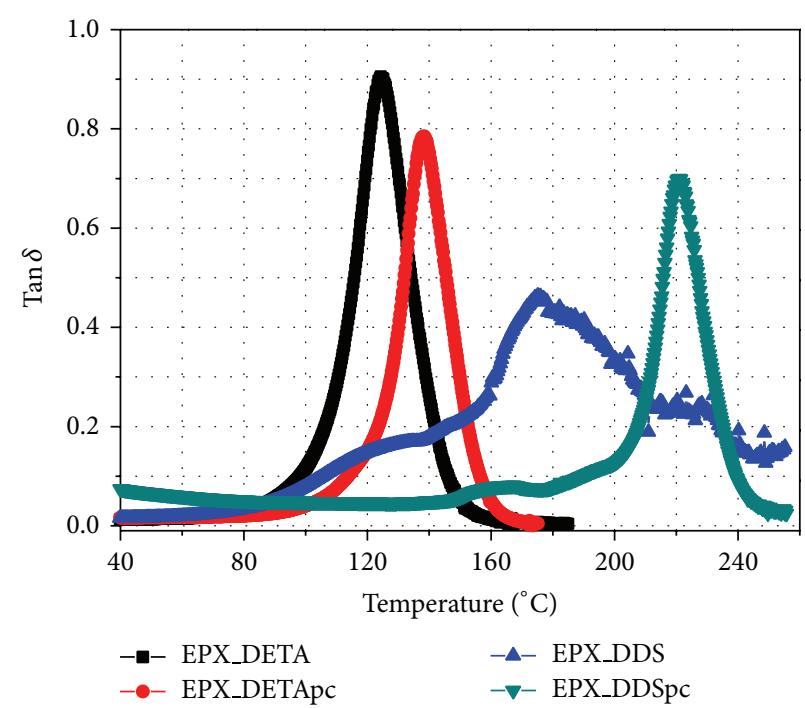

(a)

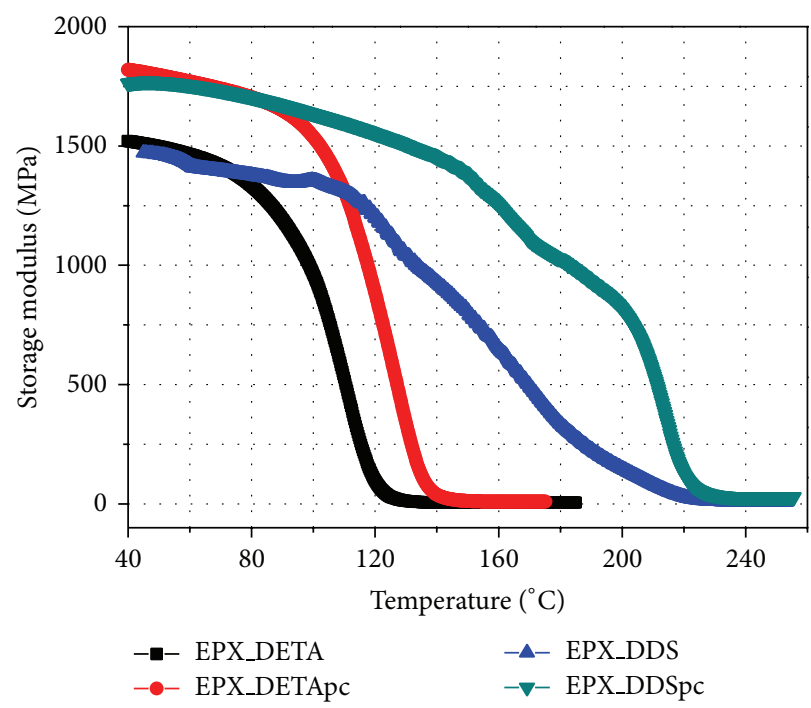

(b)

FIGURE 2: (a) Tan $\delta$ and storage modulus; (b) Tan $\delta$ as a function of the temperature for DGEBA/DDS and DGEBA/DETA epoxy system.

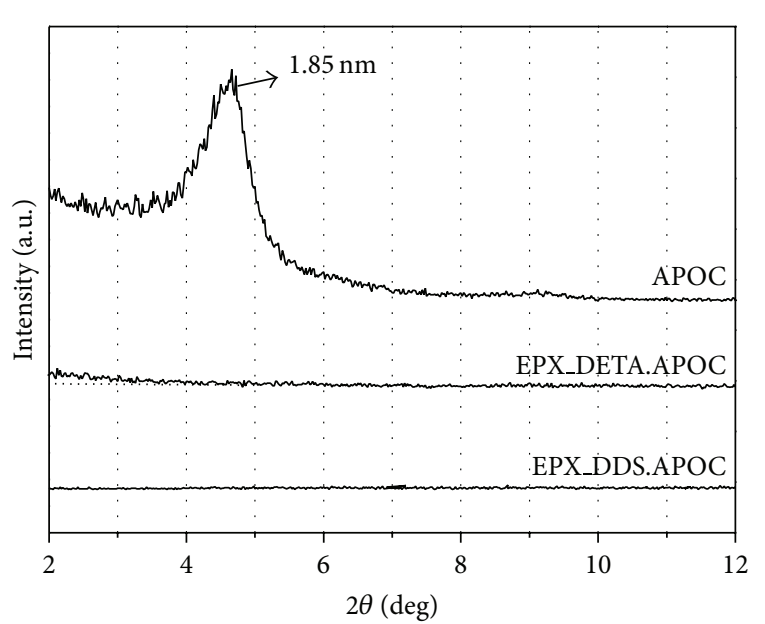

FIGURE 3: X-ray diffraction patterns of organoclay (APOC) and the nanocomposite containing 1 parts per hundred of organoclay (EPX_DDS.APOC) and (EPX_DETA.APOC) resins.

exfoliated nanocomposite EPX_DDS.APOC for the system (as the absence of the peak in the diffractogram) and is indicative of exfoliation for the EPX_DETA.APOC system (as slight inclination of $2 \theta$ around $2^{\circ}$ ). These results are similar to those reported in the literature, where the authors have shown that there is a direct correlation between the X-ray diffraction data and transmission electron microscopy (TEM); that is, absence of peak (001) in the diffractogram corresponds to a nanocomposite with exfoliated structure $[8,9,16]$.

\subsection{Thermal Mechanical Properties of the Nanocomposites.} The incorporation of only $1 \mathrm{phr}$ of organoclay in the postcured epoxy systems (EPX_DETA.APOC and EPX_DDS.APOC) resulted in an increase of the glass transition temperature $\left(T_{g}\right)$ and of the thermal stability of these systems (Figure 4(a) and Table 2). The increase in $T_{g}$ was approximately of $9^{\circ} \mathrm{C}$ when the APOC organoclay was incorporated. These results can be attributed to high aspect ratio of clay dispersion in polymer matrix that can act as physical crosslinker, whereas the epoxy resin was intercalated in the galleries of the clay, forming a predominantly exfoliated structure, as XRD data shown in Figure 3. It is also observed that the $T_{g}$ of the system EPX_DDS.APOC was considerably higher $\left(72^{\circ} \mathrm{C}\right)$ than the system EPX_DETA.APOC probably due to greater exfoliation of clay layers in polymer matrix.

Note that the nanocomposites are more thermally stable than the pure epoxy systems, especially the nanocomposite EPX_DDS.APOC, as indicated by higher plateau modulus vitreous and a lower percentage of loss modulus with temperature (Figure 4 and Table 3). These results can be attributed to restricted mobility of polymer chains due to high aspect ratio and high stiffness of the silicate layers dispersed in the polymer matrix. A significant improvement in storage modulus between 40 and $90^{\circ} \mathrm{C}$ was also portrayed in other works $[16,17]$.

According to Figure 4(b) the increases in storage modulus $E^{\prime}$ of exfoliated nanocomposites were more pronounced in the glassy region $\left(T<T_{g}\right)$, indicating that the organoclay nanolayers were well exfoliated and dispersed homogeneously in the polymer matrix, especially for system cured with DDS [11]. This may have contributed to the formation of strong interaction forces between the epoxy resin and organoclay as the epoxy polymer chains are trapped in the clay surface through hydrogen bonds, due to the interactions between the hydroxyl groups of the organoclay and groups of ether epoxy resin, as shown in Figure 5 [16].

In summary, the thermomechanical properties observed in the epoxy cured with DDS hardener proved to be useful when used as matrix and Ni-Ti shape memory alloy (SMA) which are embedded in the nanocomposite, since it has both 


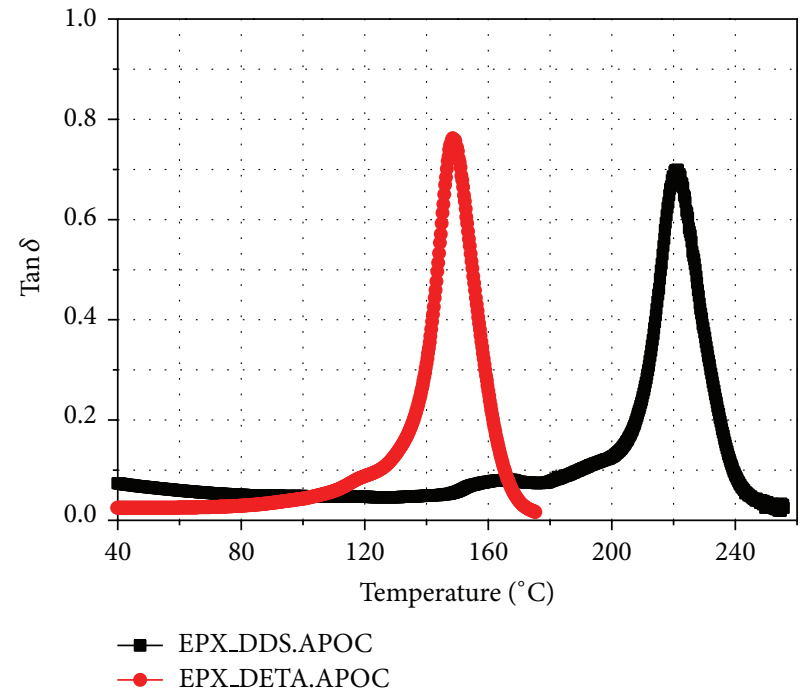

(a)

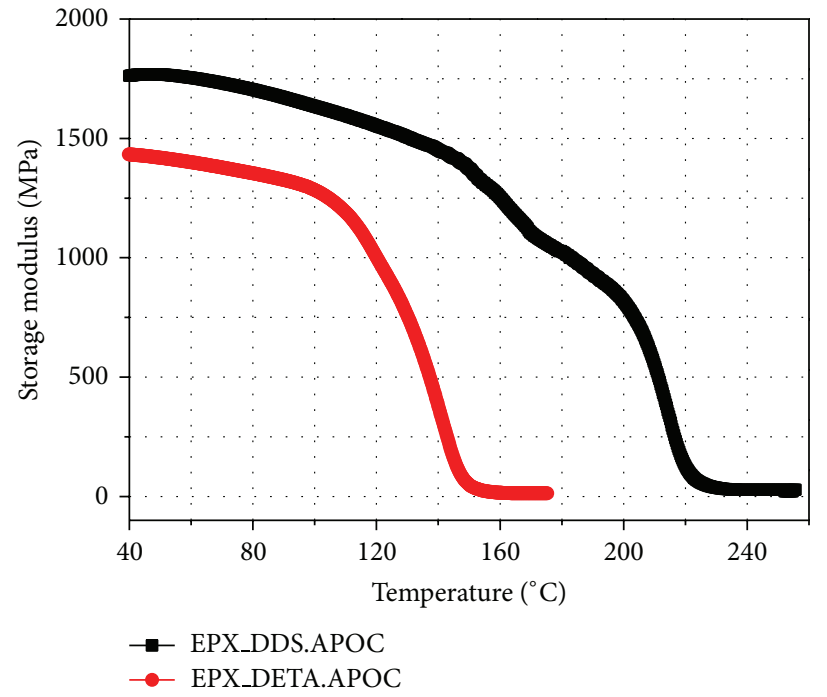

(b)

Figure 4: (a) Tan $\delta$ and storage modulus; (b) Tan $\delta$ as a function of the temperature for DGEBA/DDS and DGEBA/DETA epoxy system.

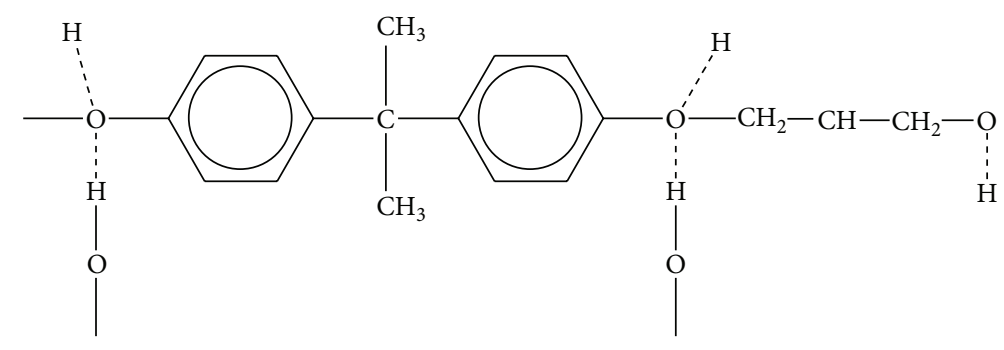

Clay layer

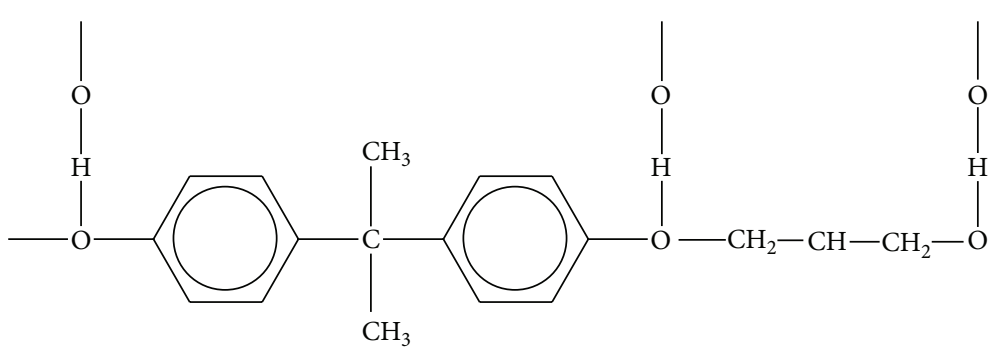

FIGURE 5: Interactions of hydrogen bonds between DGEBA and epoxy clay layer.

TABLE 3: Storage modulus $\left(E^{\prime}\right)$ and $T_{g}(\tan \delta$ peak) for different epoxy systems.

\begin{tabular}{lcccccrr}
\hline \multirow{2}{*}{ Epoxy system } & \multicolumn{9}{c}{$E^{\prime}(\mathrm{GPa})$} & \multicolumn{2}{c}{${ }^{\circ}$ lost of $E^{\prime}$} & $T_{g}\left({ }^{\circ} \mathrm{C}\right)$ \\
\hline EPX_DETApc & $40^{\circ} \mathrm{C}$ & $50^{\circ} \mathrm{C}$ & $60^{\circ} \mathrm{C}$ & $70^{\circ} \mathrm{C}$ & $80^{\circ} \mathrm{C}$ & $90^{\circ} \mathrm{C}$ & 140.52 \\
EPX_DDSpc & 1.82 & 1.79 & 1.77 & 1.74 & 1.70 & 1.64 & 9.89 \\
EPX_DETA.APOC & 1.33 & 1.30 & 1.29 & 1.25 & 1.23 & 1.21 & 9.02 \\
EPX_DDS.APOC & 1.43 & 1.41 & 1.40 & 1.37 & 1.35 & 1.32 & 7.69 \\
\hline
\end{tabular}


$T_{g}$ and thermal stability high enough to withstand repeated heating of SMA wires in the range of transformation phase, and the transformation temperatures of this type of material are found in regions near the temperature where the matrix is thermally stable.

\section{Conclusions}

The thermal behavior of the epoxy systems investigated was affected by thermal treatment of postcuring, especially when bentonite purification is used. XRD analysis showed exfoliation in the organoclay nanolayers for nanocomposite EPX_DDS.APOC and was indicative of exfoliation for the system EPX_DETA.APOC. Thermomechanical tests (DMA) indicated that EPX_DDS.APOC nanocomposites present higher $T_{g}$ and thermal stability, compared with pure EPX_DETA, EPX_DDS, and EPX_DETA.APOC. Thus, the system EPX_DDS.APOC can be used in future work or preparation of nanocomposites activated with shape memory alloys (SMA) since the transformation temperature of SMA occurs approximately at the temperature where the matrix is thermally stable, that is, between 40 and $80^{\circ} \mathrm{C}$.

\section{Conflict of Interests}

The authors declare that there is no conflict of interests regarding the publication of this paper.

\section{Acknowledgments}

The authors thank CNPQ, CAPES, and FINEP by financial support and to the researchers cited in the text that helped in the improvement of this work.

\section{References}

[1] L. A. Utracki, Clay-Containing Polymeric Nanocomposites, vol. 1-2, Rapra Technology Limited, London, UK, 2004.

[2] S. Sinha Ray and M. Okamoto, "Polymer/layered silicate nanocomposites: a review from preparation to processing," Progress in Polymer Science, vol. 28, no. 11, pp. 1539-1641, 2003.

[3] T. Lan, P. D. Kaviratna, and T. J. Pinnavaia, "Mechanism of clay tactoid exfoliation in epoxy-clay nanocomposites," Chemistry of Materials, vol. 7, no. 11, pp. 2144-2150, 1995.

[4] J. Massam and T. J. Pinnavaia, MRS Symposia Proceedings, Materials Research Society, Pittsburgh, Pa, USA, 1998.

[5] R. G. S. Santos, Study of effect on chemorheology of nanostructured organophilic clay epoxy resin composite due to filler-matrix interaction [M.S. thesis], Escola de Engenharia de Lorena, Universidade de São Paulo, Lorena, Brazil, 2009.

[6] J. Lee, T. Takekoshi, and E. Giannelis, "Fire retardant polyetherimide nanocomposites," in Nanophase and Nanocomposite Materials II, S. Komarneni, J. C. Parker, and H. J. Wollenberger, Eds., vol. 457 of MRS Proceedings, pp. 513-518, Materials Research Society, Pittsburgh, Pa, USA, 1998.

[7] D. Ratna, N. R. Manoj, R. Varley, R. K. Singh Raman, and G. P. Simon, "Clay-reinforced epoxy nanocomposites," Polymer International, vol. 52, no. 9, pp. 1403-1407, 2003.
[8] A. Yasmin, J. L. Abot, and I. M. Daniel, "Processing of clay/ epoxy nanocomposites by shear mixing," Scripta Materialia, vol. 49, no. 1, pp. 81-86, 2003.

[9] K. Wang, L. Chen, M. Kotaki, and C. He, "Preparation, microstructure and thermal mechanical properties of epoxy/crude clay nanocomposites," Composites Part A: Applied Science and Manufacturing, vol. 38, no. 1, pp. 192-197, 2007.

[10] A. Asif, R. V. Lakshmana, V. Saseendran, and K. N. Ninan, "Thermoplastic toughened layered silicate epoxy ternary nanocomposites-preparation, morphology, and thermomechanical properties," Polymer Engineering and Science, vol. 10, pp. 1002-1017, 2009.

[11] R. Kotsilkova, V. Petkova, and J. Pelovski, Thermoset Nanocomposites for Engineering Applications, Smithers Rapra Technology Limited, 2007.

[12] I. F. Leite, A. P. S. Soares, L. H. Carvalho, C. M. O. Raposo, O. M. L. Malta, and S. M. L. Silva, "Characterization of pristine and purified organobentonites," Journal of Thermal Analysis and Calorimetry, vol. 100, no. 2, pp. 563-569, 2010.

[13] Y. Zhou, F. Pervin, M. A. Biswas, V. K. Rangari, and S. Jeelani, "Fabrication and characterization of montmorillonite clayfilled SC-15 epoxy," Materials Letters, vol. 60, no. 7, pp. 869-873, 2006.

[14] F. R. Jones, "Composite materials," in Chemistry and Technology of Epoxy Resins, B. Ellis, Ed., pp. 256-302, Chapman \& Hall, London, UK, 1993.

[15] R. Velmurugan and T. P. Mohan, "Room temperature processing of epoxy-clay nanocomposites," Journal of Materials Science, vol. 39, no. 24, pp. 7333-7339, 2004.

[16] X. Kornmann, H. Lindberg, and L. A. Berglund, "Synthesis of epoxy-clay nanocomposites. Influence of the nature of the curing agent on structure," Polymer, vol. 42, no. 10, pp. 44934499, 2001.

[17] W.-B. Xu, S.-P. Bao, and P.-S. He, "Intercalation and exfoliation behavior of epoxy resin/curing agent/montmorillonite nanocomposite," Journal of Applied Polymer Science, vol. 84, no. 4, pp. 842-849, 2002. 

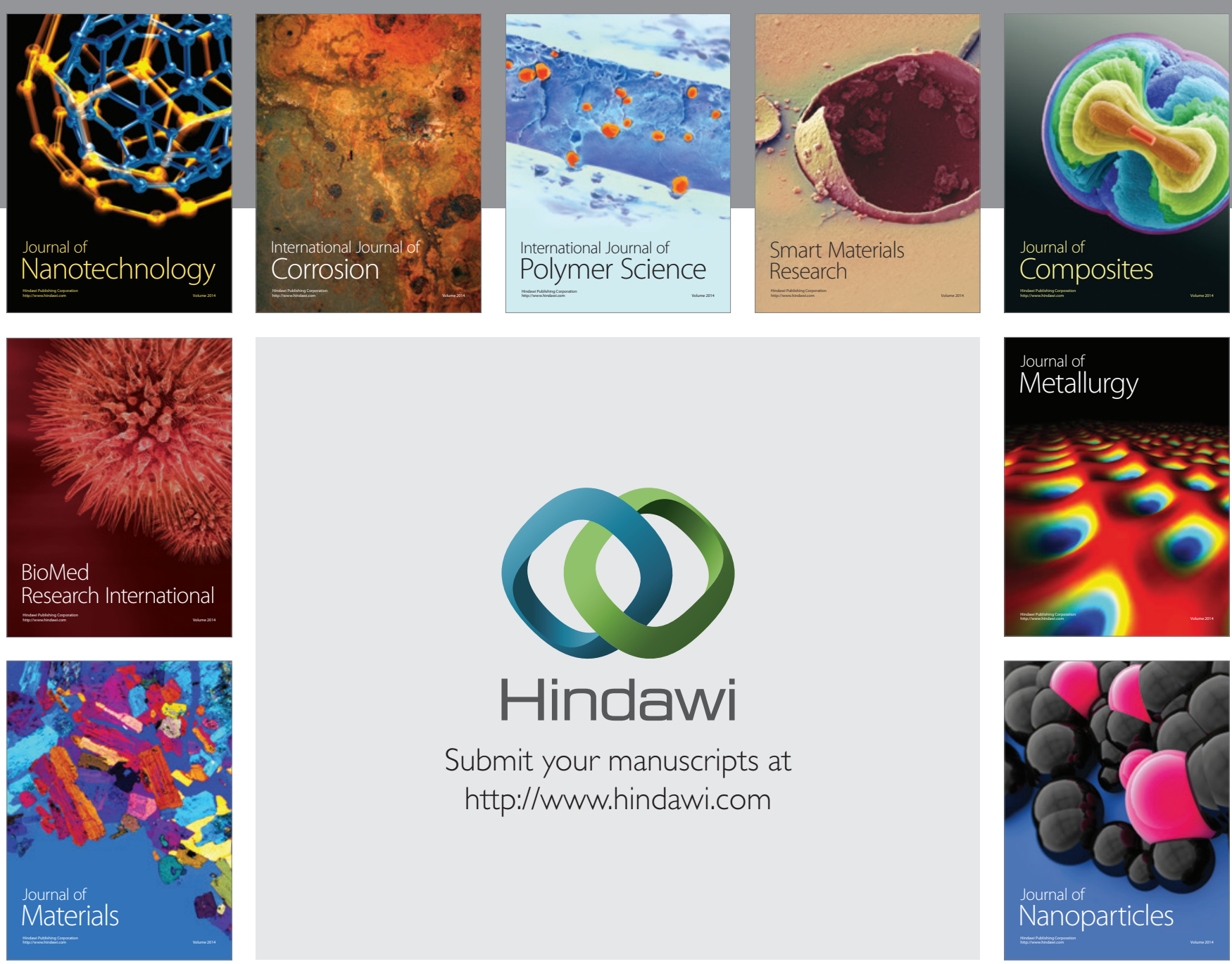

Submit your manuscripts at http://www.hindawi.com
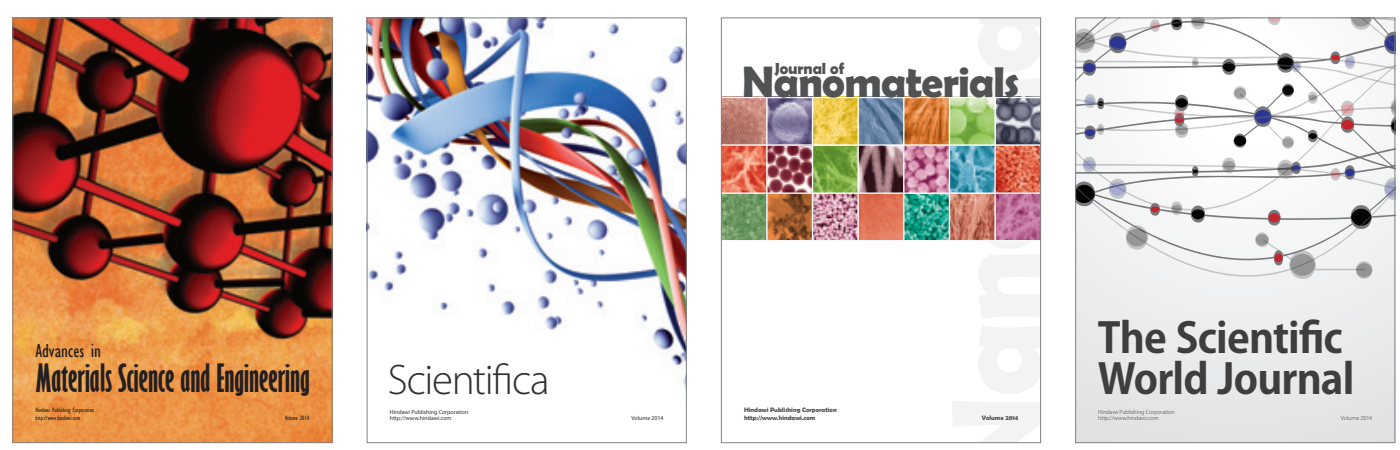

\section{The Scientific World Journal}
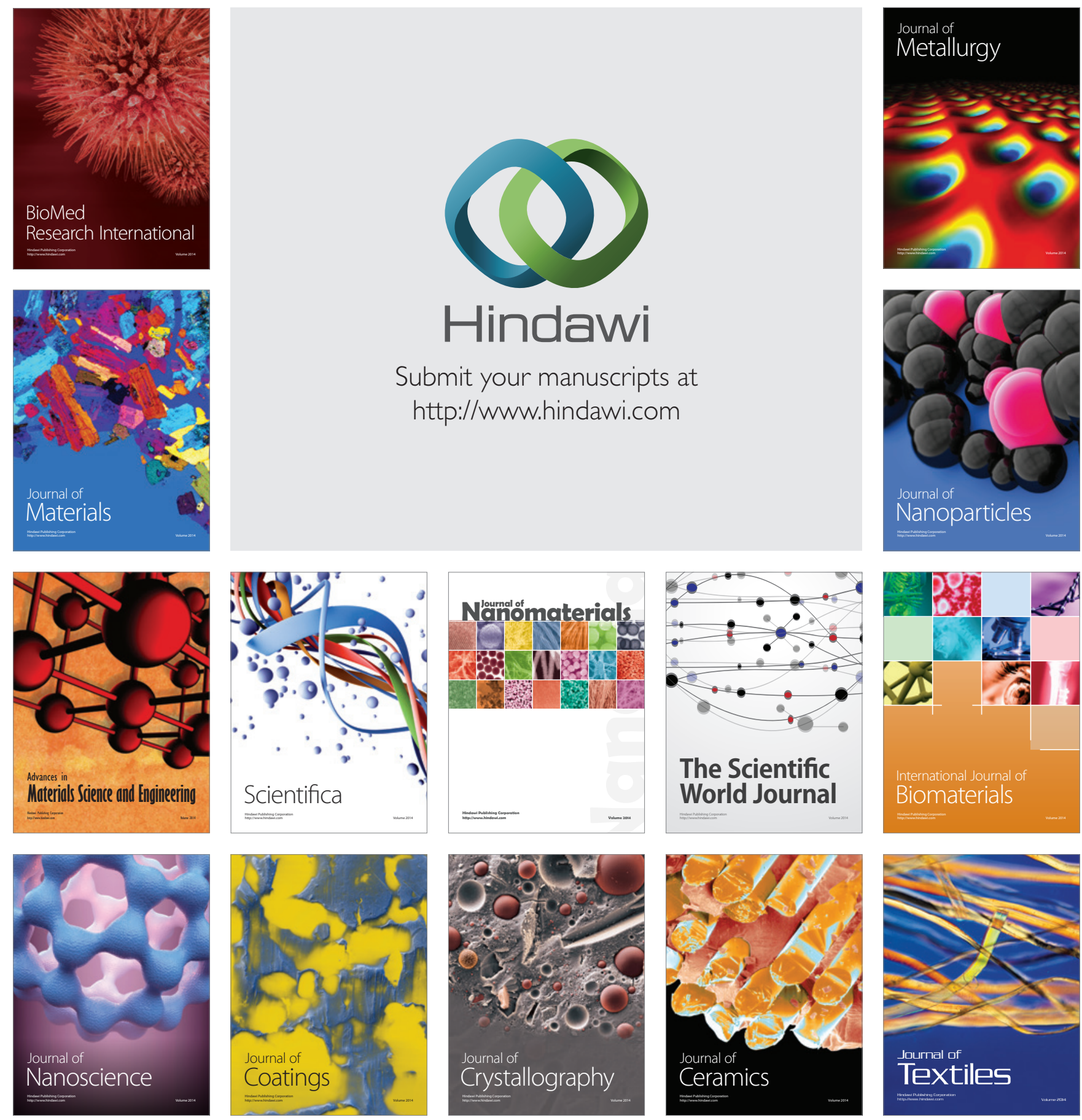